\title{
The relationship between PLOD1 expression level and glioma prognosis investigated using public databases
}

\author{
Lei Tian ${ }^{1}$, Huandi Zhou ${ }^{1}$, Guohui Wang ${ }^{1}$, Wen yan Wang ${ }^{1}$, Yuehong Li ${ }^{2}$, Xiaoying Xue ${ }^{\text {Corresp. }{ }^{1}}$ \\ 1 Department of Radiotherapy, The Second Hospital of Hebei Medical University, Shijiazhuang, Hebei, China \\ 2 Department of Pathology, The second Hospital of Hebei Medical University, Shijiazhuang, Hebei, China \\ Corresponding Author: Xiaoying Xue \\ Email address: xxy0636@163.com
}

Background. Glioma is the most common type of intracranial tumor with high malignancy and poor prognosis despite the use of various aggressive treatments. Targeted therapy and immunotherapy are not effective and new biomarkers need to be explored. Some Procollagen-lysine 2-oxyglutarate 5-dioxygenase (PLOD) family members have been found to be involved in the metastasis and progression of tumors. Both PLOD2 and PLOD3 had been reported to be highly expressed in gliomas, while the prognostic value of PLOD1 remains to be further illustrated, so we want to investigate the PLOD1 expression in glioma and its clinical implication. Methods. We collected gene expression and corresponding clinical data of glioma from the Chinese Glioma Genome Atlas (CGGA) database, The Cancer Genome Atlas (TCGA) database and Gene Expression Omnibus (GEO) database. First, we analyzed the expression and mutation of PLOD1 in gliomas and its relationship with clinicopathologic characteristics. Then, we conducted survival analysis, prognostic analysis and nomogram construction of the PLOD1 gene. Finally, we conducted gene ontology (GO) enrichment analysis and gene set enrichment analysis (GSEA) to explore possible mechanisms and gene co-expression analysis was also be performed. Results. The results showed that the expression level of PLOD1 was higher in gliomas than normal tissues, and high expression of PLOD1 was related to poor survival which can serve as an oncogenic factor and an independent prognostic indicator for glioma patients. Both the GO and GSEA analysis showed high expression of PLOD1 were enriched in Extracellular matrix (ECM) related pathways, the co-expression analysis revealed that PLOD1 was positively related to HSPG2, COL6A2, COL4A2, FN1, COL1A1, COL4A1, CD44, COL3A1, COL1A2 and $S P P 1$, and high expression of these genes were also correlated to poor prognosis of glioma. Conclusions. The results showed that high expression of PLOD1 leads to poor prognosis, and PLOD1 is an independent prognostic factor and a novel biomarker for the treatment of glioma. Furthermore, targeting PLOD1 is most likely a potential therapeutic strategy for glioma patients. 
1 The relationship between PLOD1 expression level and 2 glioma prognosis investigated using public databases

3

Lei Tian ${ }^{1}$, Huandi Zhou ${ }^{1}$, Guohui Wang ${ }^{1}$, Wenyan Wang ${ }^{1}$, Yuehong Li $^{2}$, Xiaoying Xue ${ }^{1}$

${ }^{1}$ Department of Radiotherapy, The second Hospital of Hebei Medical University, Shijiazhuang, Hebei, China

${ }^{2}$ Department of Pathology, The second Hospital of Hebei Medical University, Shijiazhuang, Hebei, China Corresponding Author: Xiaoying Xue ${ }^{1}$ Heping West Street, Shijiazhuang, Hebei, 050000, China Email address: xxy0636@163.om

\section{Abstract}

Background. Glioma is the most common type of intracranial tumor with high malignancy and poor prognosis despite the use of various aggressive treatments. Targeted therapy and immunotherapy are not effective and new biomarkers need to be explored. Some Procollagenlysine 2-oxyglutarate 5-dioxygenase ( $P L O D$ ) family members have been found to be involved in the metastasis and progression of tumors. Both PLOD2 and PLOD3 had been reported to be highly expressed in gliomas, while the prognostic value of $P L O D 1$ remains to be further illustrated, so we want to investigate the $P L O D 1$ expression in glioma and its clinical implication.

Methods. We collected gene expression and corresponding clinical data of glioma from the Chinese Glioma Genome Atlas (CGGA) database, The Cancer Genome Atlas (TCGA) database and Gene Expression Omnibus (GEO) database. First, we analyzed the expression and mutation of PLOD1 in gliomas and its relationship with clinicopathologic characteristics. Then, we conducted survival analysis, prognostic analysis and nomogram construction of the PLODI gene. Finally, we conducted gene ontology (GO) enrichment analysis and gene set enrichment analysis (GSEA) to explore possible mechanisms and gene co-expression analysis was also be performed.

Results. The results showed that the expression level of $P L O D 1$ was higher in gliomas than normal tissues, and high expression of $P L O D 1$ was related to poor survival which can serve as an oncogenic factor and an independent prognostic indicator for glioma patients. Both the GO and GSEA analysis showed high expression of PLOD1 were enriched in Extracellular matrix (ECM) related pathways, the co-expression analysis revealed that $P L O D 1$ was positively related to HSPG2, COL6A2, COL4A2, FN1, COL1A1, COL4A1, CD44, COL3A1, COL1A2 and SPP1, and high expression of these genes were also correlated to poor prognosis of glioma. Conclusions. The results showed that high expression of PLOD1 leads to poor prognosis, and $P L O D 1$ is an independent prognostic factor and a novel biomarker for the treatment of glioma. Furthermore, targeting $P L O D 1$ is most likely a potential therapeutic strategy for glioma patients. Introduction 
40 Gliomas are the most common type of primary intracranial tumor with high malignancy and poor 41 prognosis, especially in high-grade gliomas (Ostrom et al., 2020). The current standard approach 42 of treatment is maximum surgical resection followed by adjuvant radiotherapy and 43 chemotherapy (van den Bent et al. 2017; Stupp et al., 2009), and newly developed alternating 44 electric field therapy has been recommended for glioblastoma (GBM) in recent years (Sampson, 45 2015). Despite the using of multiple aggressive therapies, the overall survival of gliomas remained poor, so it is urgent to explore more effective treatment methods. In recent years, immunotherapy and targeted therapy have made great progress in many other tumors, but have little effect in glioma (Fecci \& Sampson, 2019), and although some of the molecular features showed some prognostic values (Cairncross et al., 2012; Hegi et al., 2005), none of them had became a target for targeting therapy, thus developping novel and targeted therapeutic options is urgent.

Extracellular matrix (ECM) is an important constituent of tumor microenvironment, corelating with tumor development and progression. Among the various ECM components, collagens are the most abundant proteins, and its deposition and cross-linking are closely related to tumor proliferation and invasion (Jover et al., 2018). Procollagen-lysine 2-oxyglutarate 5-dioxygenase (PLOD) catalyzed hydroxylysine residue, which is critical for the formation of covalent crosslink (Qi \& Xu, 2018). An increasing number of evidences indicate that the PLOD family, which consists of PLOD1, PLOD2 and PLOD3, plays an important role in the development and progression of tumors. Both PLOD2 and PLOD3 had been reported to be highly expressed in gliomas and were associated with tumor progression and prognosis (Song et al., 2017; Tsai et al., 2018). Some previous studies revealed that PLODI promoted tumorigenesis and metastasis in osteosarcoma, bladder cancer and esophageal squamous cell carcinoma (Wu et al., 2020; Yamada et al., 2019; Li et al., 2017), while the expression and prognostic role of PLOD1 in glioma remain to be further illustrated.

Bioinformatics analysis using high-throughput sequencing and clinical data is developing rapidly to identify sensitive biomarkers and prognostic factors for a variety of tumors, including gliomas. The acetylation modification and kinase activity of $P A K 1$ were considered to be an instrumental role in hypoxia-induced autophagy initiation and maintaining GBM growth, and PAKl might represent potential therapeutic targets for GBM treatment (Feng et al., 2020). HISTIH2BK was identified as an indicator of poor prognosis and a promising biomarker for the treatment of lowgrade glioma (LGG) (Liu et al., 2020). Similarly, $A R L 9$ had been shown its prognostic value in LGG, and probably played an important role in immune cell infiltration (Tan et al., 2020). All these markers are possible used for advanced decision-making processes in the future, however, many more potential prognostic indicators still need to be explored in gliomas. Therefore, this study attempts to analyze the PLOD1 gene expression levels in glioma and normal tissue, using public database. We also explored the relationship between PLOD1 expression and clinical characteristics as well as prognosis. Finally, we identified the PLODIrelated signaling pathways and suggested that $P L O D 1$ acted as a cancer-promoting factor in 
79

80

81

82

83

84

85

86

87

88

89

90

91

92

93

94

95

96

97

98

99

100

101

102

103

104

105

106

107

108

109

110

111

112

113

114

115

116

117

118

tumor progression, providing a potential prognostic biomarker and therapeutic target for glioma patients.

\section{Materials \& Methods}

\section{Data collection and download}

The clinical data and gene expression data were obtained from Chinese Glioma Genome Atlas (CGGA, http://www.cgga.org.cn/) database. Two datasets were downloaded containing 1018 samples (Wang et al., 2015; Liu et al., 2018; Bao et al., 2014; Zhao et al., 2017) up to May 6, 2020, and 20 non-glioma brain tissues were downloaded for analyzed. In addition, The Cancer Genome Atlas (TCGA, https://portal.gdc.cancer.gov/) database containing 592 glioma samples was collected for validation. There were 449 LGG samples and 143 GBM samples, respectively. Then, we searched "glioma" and "GEO" in the Gene Expression Omnibus (GEO, https://www.ncbi.nlm.nih.gov/geo/) database and selected GSE4290, GSE7696 and GSE50161 as validation sets (https://www.ncbi.nlm.nih.gov/geo/query/acc.cgi?acc=GSE4290, https://www.ncbi.nlm.nih.gov/geo/query/acc.cgi?acc=GSE7696, https://www.ncbi.nlm.nih.gov/geo/query/acc.cgi?acc=GSE50161). GSE4290 dataset contains 153 glioma samples and 23 normal samples (Sun et al., 2006), GSE7696 dataset contains 80 glioma samples and 4 normal samples (Lambiv et al., 2011), and GSE50161 dataset contains 117 glioma samples and 13 normal samples (Griesinger et al., 2013). Before further analysis, RNA sequencing data were $\log 2$-transformed. All these databases were screened to eliminate samples missing clinical information.

\section{Gene expression analysis, GEPIA database analysis, mutation analysis and Cancer Cell}

\section{Line Encyclopedia (CCLE) analysis}

The expression data of PLODI in normal brain tissue and glioma in CGGA dataset were imported into GraphPad Prism 8 software for analysis, and then validated by GSE4290 dataset. In addition, PLODI expression analyses of GBM and LGG were also performed in GEPIA (http://gepia.cancer-pku.cn/) (Tang et al., 2017). In addition, we performed mutation analysis to better comprehend the genomics profile of PLOD1 based on the cBioPortal online database (Gao et al., 2013). Furthermore, we used the CCLE database (https://portals.

broadinstitute.org/ccle/home) to assess $P L O D 1$ expression in different cancers.

\section{Correlations between PLOD1 expression and clinical outcomes and clinicopathologic characteristics}

The data from the CGGA datasets were mainly used to research the prognostic role of PLOD1 in gliomas by using R software. According to the median expression level of PLOD1, high and low group were divided. The "survival" and "survminer" package were used in R software to plot survival curves for different $P L O D 1$ expression level. And then, the survival data of different IDH and MGMT promoter methylation status were imported into GraphPad Prism 8 software for survival analysis, so as to explore the survival of PLOD1 expression levels in different molecular types of gliomas. We used the "survival ROC" package to calculate receiver operator characteristic (ROC) curves for PLODI at 1, 3, and 5 years using the Kaplan-Meier method. Univariate and multivariate Cox analysis were also performed to assess the 
119 predictive value of $P L O D 1$ at a significance level of $\mathrm{P}<0.001$. Based on the TCGA datasets, 120 survival curve and ROC curves were performed to validate. To develop an individual prognostic 121 signature for the 1-, 2- and 3-year survival rates, we constructed a nomogram in CGGA cohort 122 using the "survival" and the "rms" package in R software. Following that, calibration curves

123

124

125

126

127

128

129

130

131

132

133

134

135

136

137

138

139

140

141

142

143

144

145

146

147

148

149

150

151

152

153

154

155

156

157

158 were plotted to evaluate the concordance between actual and predicted survival. In addition, the correlationship between PLOD1 expression and clinicopathologic characteristics was performed using the "beeswarm" package in R software.

\section{Differential genes expression analysis and enrichment analysis for significant pathways}

The mRNA sequencing datas in glioma from CGGA datasets were normalized and the differentially expressed genes (DEGs) including significantly upregulated and downregulated genes were screened with an adjusted $p$ value $<0.05$ and absolute $\log 2$ fold change (FC) $>2$, and then a volcano plot of DEGs was generated using the "limma" package in R software (Ritchie et al., 2015). Using the screened DEGs, gene ontology (GO) enrichment analysis was performed on the online tool-Metascape (Zhou et al., 2019) (http://metas cape.org/gp/index .html\#/main/step1). In addition, gene set enrichment analysis (GSEA) was also performed to indirectly explain the function of PLOD1 (Subramanian et al., 2005). A gene set was considered as an enriched group when the NES $>1$ and FDR score $<0.05$.

\section{Co-expression analysis}

The GO and KEGG analysis both enriched in extracellular matrix (ECM). The common genes of DEGs and the key genes in the ECM pathways enriched by the KEGG analysis were performed for correlation analysis between $P L O D 1$. Pearson correlation analysis was used for parametric tests, Spearman correlation analysis was used for non-parametric tests. A circular plot and a pheatmap of the common genes positively associated with $P L O D 1$ were generated by $\mathrm{R}$ software. In addition, every common gene was preformed in GEPIA to examination the expression and survival in gliomas.

\section{Statistical analysis}

Statistical analyses were performed with R software v3.6.3 (http://www.r-project.org/) (Hjalt et al., 2001), and Prism 8 (GraphPad Software, Inc). The "survival" package (https://CRAN.Rproject.org/package=survival), "survminer" package (https://CRAN.R-

project.org/package=survminer), "survivalROC" package (https://CRAN.Rproject.org/package=survivalROC), "rms" package, "beeswarm" package (https://CRAN.Rproject.org/package=beeswarm), "limma" package, "ggplot2" package, "pheatmap" package (https://CRAN.R-project.org/package=pheatmap), "corrplot" package

(https://github.com/taiyun/corrplot), and "circlize" package (Gu et al., 2014) of R software were used successively. Data were considered significant at $\mathrm{P}<0.05$.

\section{Results}

\section{Characteristics of the samples}

The workflow of our study is shown in Fig. 1. After screening, a total of 636, 592, 153, 80 and 117 glioma patient samples were obtained from the CGGA, TCGA, GSE4290, GSE7696 and GSE50161 datasets. Both CGGA and TCGA datasets contained grading data, age, gender, IDH 
159

160

161

162

163

164

165

166

167

168

169

170

171

172

173

174

175

176

177

178

179

180

181

182

183

184

185

186

187

188

189

190

191

192

193

194

195

196

197

198

mutation status and $1 \mathrm{p} 19$ codeletion status. Additionally, postoperative radiotherapy or chemotherapy follow-up and MGMT methylation data were only included in the CGGA dataset. Detailed clinical information classification and percentages of glioma patients are shown in Table 1.

\section{$P L O D 1$ gene expression and genomic characteristics in glioma}

The expression level of PLODI was significantly higher in gliomas than in normal tissues based on the CGGA and GEO datasets (Fig. 2A, B, C, D), and the same results were obtained in GEPIA online analysis in both GBM and LGG (Fig. 2E). The genomic alteration of PLOD1 was shown in Fig. 2F, and several mutation types were provided using cBioPortal online database (Fig. 2G). In addition, the results from the CCLE database showed that PLOD1 expression in glioma ranked $4^{\text {th }}$ among the cell lines from different cancer tissues (Fig. $2 \mathrm{H}$ ).

\section{Survival analysis and prognostic values of $P L O D 1$ in glioma patients}

The Kaplan-Meier survival analysis of the CGGA datasets showed that high level expression of PLOD1 related to poor survival ( $\mathrm{p}<0.001$, Fig. $3 \mathrm{~A}$ ), and the same results were obtained by using the TCGA datasets ( $<<0.001$, Fig. 3B). Furthermore, we investigated the correlation between PLOD1 expression and IDH and MGMT promoter methylation status on survival. The results showed that patients with IDH mutation had a longer survival regardless of the level of PLODI expression ( $\mathrm{p}<0.001$, Fig. $3 \mathrm{C}$ ). However, patients with low expression of PLOD1 had a longer survival regardless of the MGMT promoter methylation status $(\mathrm{p}<0.001$, Fig. 3D). In addition, based on the CGGA datasets, ROC curve revealed that PLOD1 was a predictive marker of 1-year (AUC=0.816), 3-year (AUC=0.793), and 5-year survival (AUC=0.707) (Fig. 3E). Similarly, ROC curve analysis using TCGA datasets also verified this result. The area under curve for OS was 0.786 at 1 years, 0.805 at 3 years, and 0.796 at 5 years, respectively (Fig. 3F).

\section{Independent prognostic analysis and development of nomogram of PLOD1 in glioma}

To identify whether PLODI was an independent prognostic index, univariate and multivariate Cox regression analyses were performed in CGGA datasets. Univariate analysis showed that PLOD1 expression $(\mathrm{HR}=1.986 ; 95 \% \mathrm{CI}=1.796-2.198 ; \mathrm{P}<0.001)$, PRS type, grade, age, IDH mutation, and 1p19q codeletion were significantly associated with OS (Fig. 4A). Furthermore, multivariate Cox regression analysis revealed that $P L O D 1$ expression $(\mathrm{HR}=1.283 ; 95 \%$ $\mathrm{CI}=1.128-1.460 ; \mathrm{P}<0.001)$, PRS type, grade, chemotherapy after resection, IDH mutation, and 1p19q codeletion remained significantly correlated with OS (Fig. 4B). These results indicated that $P L O D 1$ expression has a strong prognostic value in gliomas. Furthermore, to quantitatively predict the prognosis of glioma patients, we constructed a nomogram using grade, IDH mutation status, MGMT promoter methylation status, 1p19q codeletion status and PLOD1 expression level. It revealed that the $P L O D 1$ expression level was the leading factor for predicting nomogram (Fig. 4C). Calibration curves indicated that actual and predicted survival matched very well, especially for 3-year survival (Figure. 4D-F).

\section{Correlations between $P L O D 1$ expression and clinicopathologic characteristics}

Among the analysis, patients older than 42 years had significantly higher levels of PLOD1 expression ( $\mathrm{p}<0.001$, Fig. 5A). The expression of PLOD1 increased with the increase of glioma 
199

200

201

202

203

204

205

206

207

208

209

210

211

212

213

214

215

216

217

218

219

220

221

222

223

224

225

226

227

228

229

230

231

232

233

234

235

236

237

238

grade ( $\mathrm{p}<0.001$, Fig. $5 \mathrm{~B})$ and was higher in recurrent and secondary tumor than primary tumor $(\mathrm{p}<0.001$, Fig. 5C). For molecular type, PLOD1 expressed lower in patients with $1 \mathrm{p} 19 \mathrm{q}$ codeletion and IDH1 mutants ( $\mathrm{p}<0.001$, Fig. 5D, E).

\section{DEGs and enrichment pathways analysis of PLOD1}

A total of 67 DEGs were identified in analysis, which included 61 upregulated and 6 downregulated genes (Fig. 6A). Metascape analysis demonstrated that the biological processes of these DEGs were significantly enriched in extracellular structure organization (Fig. 6B). In addition, GSEA was used to identify hallmarks of glioma. The results showed that ECM receptor interaction was significantly enriched in high-expression PLOD1 phenotypes (NES=1.96, normalized $\mathrm{P}=0.002$ ) (Fig. 6C). As Metascape analysis and GSEA analysis both enriched in ECM related pathways, we screened out the core genes of ECM-related pathways enriched in GSEA analysis and intersected the core genes with DEGs to obtain 10 common genes (Fig. 6D).

\section{Co-expression analysis of $P L O D 1$}

Through gene co-expression analysis, we found that PLOD 1 was closely related to all of the screened common genes. The PLOD1 was positively associated with HSPG2, COL6A2, COL4A2, FN1, COL1A1, COL4A1, CD44, COL3A1, COL1A2, SPP1 (Fig. 7A-J). A heatmap of the intersection genes associated with $P L O D 1$ was plotted (Fig. 7K), and a circular plot of these genes was also generated (Fig. 7L). Furthermore, we performed gene expression and survival analysis of the the common genes in GEPIA, the results showed that all the genes were highly expressed in glioma and high expression level related to poor prognosis (Fig. 8A-T).

\section{Discussion}

Glioma, especially high-grade glioma, has a poor prognosis due to its aggressive growth and high recurrence rate. The standard treatment for glioma is a combination of traditional surgery, radiotherapy and chemotherapy (Buckner et al., 2016; Jaeckle et al., 2020; Herrlinger et al., 2019). Emerging alternating electric field therapy has been included in the NCCN guidelines in recent years due to its significant survival benefits in glioblastoma (Stupp et al., 2017). However, the prognosis is still poor, and the choice of multiple treatment options is a clinical challenge. Therefore, it is important to explore potential biomarkers and therapeutic targets of glioma. In this study, we found that PLODI expression in glioma was higher than that in normal tissues using the CGGA datasets, which was verified by the GEO datasets and GEPIA. In addition, the expression of PLOD1 in glioma was higher than in most cancers based on the CCLE database. Previous studies have revealed that $P L O D 2$ and $P L O D 3$ have predictive effects on the prognosis of glioma (Song et al., 2017; Tsai et al., 2018), we speculated that PLOD1 also influence the prognosis of glioma, although it has not been explored. The sequential Kaplan-Meier survival analysis showed high expression level of PLOD1 related to poor prognosis based on both the CGGA and TCGA datasets. We also found that patients with different IDH mutation and MGMT promoter methylation status had different survival at different $P L O D 1$ expression levels. Through comprehensive univariate and multivariate Cox analysis, we found that PLOD1 was an independent prognostic factor in glioma patients. Moreover, the AUC values of ROC curves for 
239

240

241

242

243

244

245

246

247

248

249

250

251

252

253

254

255

256

257

258

259

260

261

262

263

264

265

266

267

268

269

270

271

272

273

274

275

276

277

278

PLOD1 at 1,3 , and 5 years were all $>0.7$, which also suggested that $P L O D 1$ was a predictor of survival. All of the above results confirmed our previous hypothesis. According to these prognostic signatures, we constructed a nomogram to quantitatively predict the survival of glioma patients, and the results showed that the PLOD1 expression level was the leading factor. We can use this model to predict the survival of glioma patients. Therefore, it can help to make clinical decisions for patients, which can avoid overtreatment or undertreatment, so as to individually select the best treatment strategies for glioma patients.

ECM is an important component of tumor microenvironment and plays an important role in cancer development and progression (Mohan, Das \& Sagi, 2020; Lu, Weaver \& Werb, 2012). Collagen is a major component of ECM, and its elevated deposition and cross-linking can worsen tumor progression depending on the hydroxylation of lysine residues, which is mainly catalyzed by PLODs (Gjaltema \& Bank, 2017; Qi \& Xu, 2018). In this study, both GO and GSEA analysis were performed to suggest that PLOD1 was enriched in ECM-related pathways, which was consistent with its pathophysiological mechanism. Using cBioPortal online analysis, we found that the mutation frequency of PLOD1 is not high (1\%), which suggests that the aberrant expression of $P L O D 1$ may be a result of post-transcriptional regulations or translation modifications. Previous studies reported that the $P L O D$ expression was mainly regulated at the transcription level (Gjaltema et al., 2015). Compared with PLOD2, the regulation of PLOD1 expression has not been well investigated. Some preliminary studies have shown that PITX2 can directly regulate $P L O D 1$ expression by binding to the promoter region, using chromatin immunoprecipitation and luciferase reporting experiments (Hjalt, Amendt \& Murray, 2001), and both $B M P-2$ and $T G F-\beta 1$ can induce $P L O D 1$ expression in adipose tissue-derived mesenchymal stem cells (Knippenberg et al., 2009). However, the mechanism of its expression regulation still needs more exploration to identify.

As we found in glioma, increased expression of PLODI is present in many types of cancer, and the high expression leads to short disease-related survival (Wu et al., 2020; Yamada et al., 2019; $\mathrm{Li}$ et al., 2017). Therefore, targeting PLOD1 is a potential therapeutic strategy, while there is no potent $P L O D 1$ inhibitor available. So, it is of great significance to explore specific inhibitors of $P L O D 1$ for preventing tumor progression. In addition, another potential strategy is to reduce $P L O D 1$ expression, this means that further understanding of the regulatory mechanism of $P L O D 1$ in the development of cancer may lead to the exploration of novel signaling pathways to target PLODI.

Finally, we screened out the common genes between the core genes of ECM-related pathways and DEGs, and then performed co-expression analysis with PLOD1. We found that PLODI was positively related to HSPG2, COL6A2, COL4A2, FN1, COL1A1, COL4A1, CD44, COL3A1, $C O L 1 A 2, S P P 1$, suggesting that they jointly contribute to the occurrence and development of gliomas. Moreover, based on GEPIA online analysis, all of them were highly expressed in glioma and its expression levels were closely related with patients' survival, therefore, it is of great significance to carry out more studies on these genes in glioma in the future.

\section{Conclusions}


279 To analyze the relationship between the expression level of PLOD1 and the prognosis of glioma, 280 we use the CGGA, TCGA and GEO datasets performing bioinformatics analysis. The results 281 showed that the expression level of PLODl was higher in glioma than normal tissues and high 282 expression of $P L O D 1$ was related to poor survival which can serve as an independent prognostic

283

284

285

286

287

288

289

290

291

292

293

294

295

296

297

298

299

300

301

302

303

304

305

306

307

308

309

310

311

312

313

314

315

316

317 indicator for glioma patients. Additionally, the GO and GSEA analysis verified that the mechanism of PLODI's oncogenic effect was related to ECM, the co-expression analysis revealed that $P L O D 1$ was positively correlated with $H S P G 2, C O L 6 A 2, C O L 4 A 2, F N 1, C O L 1 A 1$, COL4A1, CD44, COL3A1, COL1A2 and SPP1. All of them were ECM related genes and the expression of these genes was also correlated with the prognosis of glioma. In conclusion, this study indicated that targeting PLOD1 is a potential therapeutic strategy for glioma patients, and the expression level of PLOD1 may provide a reference for the selection of treatment regimens for glioma patients, which suggests that the biological functions and mechanisms of PLOD1 need to be explored in the future. However, this study mainly relies on bioinformatics analysiswith certain limitations and more in vitro or in vivo validation experiments can make it more reliable.

\section{Acknowledgements}

Thanks to all the researchers and staff working for The Cancer Genome Atlas database, The Cancer Genome Atlas database and Gene Expression Omnibus database.

\section{References}

Bao ZS, Chen HM, Yang MY, Zhang CB, Yu K, Ye WL, Hu BQ, Yan W, Zhang W, Akers J, Ramakrishnan V, Li J, Carter B, Liu YW, Hu HM, Wang Z, Li MY, Yao K, Qiu XG, Kang CS, You YP, Fan XL, Song WS, Li RQ, Su XD, Chen CC, Jiang T. 2014. RNA-seq of 272 gliomas revealed a novel, recurrent PTPRZ1-MET fusion transcript in secondary glioblastomas. Genome research 24:1765-73. DOI: 10.1101/gr.165126.113.

Buckner JC, Shaw EG, Pugh SL, Chakravarti A, Gilbert MR, Barger GR, Coons S, Ricci P, Bullard D, Brown PD, Stelzer K, Brachman D, Suh JH, Schultz CJ, Bahary JP, Fisher BJ, Kim H, Murtha AD, Bell EH, Won M, Mehta MP, Curran WJ Jr. 2016. Radiation plus Procarbazine, CCNU, and Vincristine in Low-Grade Glioma. The New England journal of medicine 374:1344-55. DOI: 10.1056/NEJMoa1500925.

Cairncross G, Wang M, Shaw E, Jenkins R, Brachman D, Buckner J, Fink K, Souhami L, Laperriere N, Curran W, Mehta M. 2012. Phase III trial of chemoradiotherapy for anaplastic oligodendroglioma: long-term results of RTOG 9402. Journal of clinical oncology: official journal of the American Society of Clinical Oncology 31:337-43. DOI: 10.1200/JCO.2012.43.2674.

Fecci PE, Sampson JH. 2019. The current state of immunotherapy for gliomas: an eye toward the future. Journal of neurosurgery 131:657-666. DOI: 10.3171/2019.5.JNS181762.

Feng X, Zhang H, Meng L, Song H, Zhou Q, Qu C, Zhao P, Li Q, Zou C, Liu X, Zhang Z. 2020. Hypoxia-induced acetylation of PAK1 enhances autophagy and promotes brain tumorigenesis via phosphorylating ATG5 1-20. DOI: 10.1080/15548627.2020.1731266. 
318 Gao J, Aksoy BA, Dogrusoz U, Dresdner G, Gross B, Sumer SO, Sun Y, Jacobsen A, Sinha R, 319 Larsson E, Cerami E, Sander C, Schultz N. 2013. Integrative analysis of complex cancer

320

321

322

323

324

325

326

327

328

329

330

331

332

333

334

335

336

337

338

339

340

341

342

343

344

345

346

347

348

349

350

351

352

353

354

355

356 genomics and clinical profiles using the cBioPortal. Science signaling 6:p11. DOI: 10.1126/scisignal.2004088.

Gjaltema RA, Bank RA. 2017. Molecular insights into prolyl and lysyl hydroxylation of fibrillar collagens in health and disease. Critical reviews in biochemistry and molecular biology 52:74-95. DOI: 10.1080/10409238.2016.1269716.

Gjaltema RA, de Rond S, Rots MG, Bank RA. 2015. Procollagen Lysyl Hydroxylase 2 Expression Is Regulated by an Alternative Downstream Transforming Growth Factor $\beta-1$ Activation Mechanism. The Journal of biological chemistry 290:28465-76. DOI: 10.1074/jbc.M114.634311.

Griesinger AM, Birks DK, Donson AM, Amani V, Hoffman LM, Waziri A, Wang M, Handler MH, Foreman NK. 2013. Characterization of distinct immunophenotypes across pediatric brain tumor types. Journal of immunology 191:4880-8. DOI: 10.4049/jimmunol.1301966.

Gu Z, Gu L, Eils R, Schlesner M, Brors B. 2014. circlize Implements and enhances circular visualization in R. Bioinformatics (Oxford, England) 30:2811-2. DOI: 10.1093/bioinformatics/btu393.

Hegi ME, Diserens AC, Gorlia T, Hamou MF, de Tribolet N, Weller M, Kros JM, Hainfellner JA, Mason W, Mariani L, Bromberg JE, Hau P, Mirimanoff RO, Cairncross JG, Janzer RC, Stupp R. 2005. MGMT gene silencing and benefit from temozolomide in glioblastoma. The New England journal of medicine 352:997-1003. DOI: 10.1056/NEJMoa043331.

Herrlinger U, Tzaridis T, Mack F, Steinbach JP, Schlegel U, Sabel M, Hau P, Kortmann RD, Krex D, Grauer O, Goldbrunner R, Schnell O, Bähr O, Uhl M, Seidel C, Tabatabai G, Kowalski T, Ringel F, Schmidt-Graf F, Suchorska B, Brehmer S, Weyerbrock A, Renovanz M, Bullinger L, Galldiks N, Vajkoczy P, Misch M, Vatter H, Stuplich M, Schäfer N, Kebir S, Weller J, Schaub C, Stummer W, Tonn JC, Simon M, Keil VC, Nelles M, Urbach H, Coenen M, Wick W, Weller M, Fimmers R, Schmid M, Hattingen E, Pietsch T, Coch C, Glas M; Neurooncology Working Group of the German Cancer Society. 2019. Lomustinetemozolomide combination therapy versus standard temozolomide therapy in patients with newly diagnosed glioblastoma with methylated MGMT promoter (CeTeG/NOA-09): a randomised, open-label, phase 3 trial. Lancet (London, England) 393:678-688. DOI: 10.1016/S0140-6736(18)31791-4.

Hjalt TA, Amendt BA, Murray JC. 2001. PITX2 regulates procollagen lysyl hydroxylase (PLOD) gene expression: implications for the pathology of Rieger syndrome. The Journal of cell biology 152:545-52. DOI: 10.1083/jcb.152.3.545.

Jaeckle KA, Ballman KV, van den Bent M, Giannini C, Galanis E, Brown PD, Jenkins RB, Cairncross JG, Wick W, Weller M, Aldape KD, Dixon JG, Anderson SK, Cerhan JH, Wefel JS, Klein M, Grossman SA, Schiff D, Raizer JJ, Dhermain F, Nordstrom DG, Flynn PJ, Vogelbaum MA. 2020. CODEL: Phase III study of RT, RT + Temozolomide (TMZ), or 
357

358

359

360

361

362

363

364

365

366

367

368

369

370

371

372

373

374

375

376

377

378

379

380

381

382

383

384

385

386

387

388

389

390

391

392

393

394

395

TMZ for newly-diagnosed 1p/19q Codeleted Oligodendroglioma. Analysis from the initial study design. Neuro-oncology noaa168. DOI: 10.1093/neuonc/noaa168.

Jover E, Silvente A, Marín F, Martínez-González J, Orriols M, Martinez CM, Puche CM, Valdés M, Rodriguez C, Hernández-Romero D. 2018. Inhibition of enzymes involved in collagen cross-linking reduces vascular smooth muscle cell calcification. FASEB journal: official publication of the Federation of American Societies for Experimental Biology 32:44594469. DOI: 10.1096/fj.201700653R.

Knippenberg M, Helder MN, Doulabi BZ, Bank RA, Wuisman PI, Klein-Nulend J. 2009. Differential effects of bone morphogenetic protein-2 and transforming growth factor-beta 1 on gene expression of collagen-modifying enzymes in human adipose tissue-derived mesenchymal stem cells. Tissue engineering. Part A 15:2213-25. DOI: 10.1089/ten.tea.2007.0184.

Lambiv WL, Vassallo I, Delorenzi M, Shay T, Diserens AC, Misra A, Feuerstein B, Murat A, Migliavacca E, Hamou MF, Sciuscio D, Burger R, Domany E, Stupp R, Hegi ME. 2011. The Wnt inhibitory factor 1 (WIF1) is targeted in glioblastoma and has a tumor suppressing function potentially by induction of senescence. Neuro-oncology 13:736-747. DOI: 10.1093/neuonc/nor036.

Li L, Wang W, Li X, Gao T. 2017. Association of ECRG4 with PLK1, CDK4, PLOD1 and PLOD2 in esophageal squamous cell carcinoma. American journal of translational research 9:3741-3748.

Liu W, Xu Z, Zhou J, Xing S, Li Z, Gao X, Feng S, Xiao Y. 2020. High Levels of HIST1H2BK in Low-Grade Glioma Predicts Poor Prognosis: A Study Using CGGA and TCGA Data. Frontiers in oncology 10:627. DOI: 10.3389/fonc.2020.00627.

Liu X, Li Y, Qian Z, Sun Z, Xu K, Wang K, Liu S, Fan X, Li S, Zhang Z, Jiang T, Wang Y. 2018. A radiomic signature as a non-invasive predictor of progression-free survival in patients with lower-grade gliomas. NeuroImage. Clinica 20:1070-1077. DOI: 10.1016/j.nicl.2018.10.014.

Lu P, Weaver VM, Werb Z. 2012. The extracellular matrix: a dynamic niche in cancer progression. The Journal of cell biology 196:395-406. DOI: 10.1083/jcb.201102147.

Mohan V, Das A, Sagi I. 2020. Emerging roles of ECM remodeling processes in cancer. Seminars in cancer biology 62:192-200. DOI: 10.1016/j.semcancer.2019.09.004.

Ostrom QT, Patil N, Cioffi G, Waite K, Kruchko C, Barnholtz-Sloan JS. 2020. CBTRUS Statistical Report: Primary Brain and Other Central Nervous System Tumors Diagnosed in the United States in 2013-2017. Neuro-oncology 22(12 Suppl 2): iv1-iv96. DOI: 10.1093/neuonc/noaa200.

Qi Y, Xu R. 2018. Roles of PLODs in Collagen Synthesis and Cancer Progression. Frontiers in cell and developmental biology 6:66. DOI: 10.3389/fcell.2018.00066.

Qi Y, Xu R. 2018. Roles of PLODs in Collagen Synthesis and Cancer Progression. Frontiers in cell and developmental biology 6:66. DOI: 10.3389/fcell.2018.00066. 
396

397

398

399

400

401

402

403

404

405

406

407

408

409

410

411

412

413

414

415

416

417

418

419

420

421

422

423

424

425

426

427

428

429

430

431

432

433

434

435

Ritchie ME, Phipson B, Wu D, Hu Y, Law CW, Shi W, Smyth GK. 2015. limma powers differential expression analyses for RNA-sequencing and microarray studies. Nucleic acids research 43:e47. DOI: 10.1093/nar/gkv007.

Sampson JH. 2015. Alternating Electric Fields for the Treatment of Glioblastoma. JAMA 314(23):2511-3. DOI: 10.1001/jama.2015.16701.

Song Y, Zheng S, Wang J, Long H, Fang L, Wang G, Li Z, Que T, Liu Y, Li Y, Zhang X, Fang W, Qi S. 2017. Hypoxia-induced PLOD2 promotes proliferation, migration and invasion via PI3K/Akt signaling in glioma. Oncotarget 8:41947-41962. DOI:

10.18632/oncotarget.16710.

Stupp R, Hegi ME, Mason WP, van den Bent MJ, Taphoorn MJ, Janzer RC, Ludwin SK, Allgeier A, Fisher B, Belanger K, Hau P, Brandes AA, Gijtenbeek J, Marosi C, Vecht CJ, Mokhtari K, Wesseling P, Villa S, Eisenhauer E, Gorlia T, Weller M, Lacombe D, Cairncross JG, Mirimanoff RO; European Organisation for Research and Treatment of Cancer Brain Tumour and Radiation Oncology Groups; National Cancer Institute of Canada Clinical Trials Group. 2009. Effects of radiotherapy with concomitant and adjuvant temozolomide versus radiotherapy alone on survival in glioblastoma in a randomised phase III study: 5-year analysis of the EORTC-NCIC trial. The Lancet. Oncology 10:459-66. DOI: 10.1016/S1470-2045(09)70025-7.

Stupp R, Taillibert S, Kanner A, Read W, Steinberg D, Lhermitte B, Toms S, Idbaih A, Ahluwalia MS, Fink K, Di Meco F, Lieberman F, Zhu JJ, Stragliotto G, Tran D, Brem S, Hottinger A, Kirson ED, Lavy-Shahaf G, Weinberg U, Kim CY, Paek SH, Nicholas G, Bruna J, Hirte H, Weller M, Palti Y, Hegi ME, Ram Z. 2017. Effect of Tumor-Treating Fields Plus Maintenance Temozolomide vs Maintenance Temozolomide Alone on Survival in Patients With Glioblastoma: A Randomized Clinical Trial 318:2306-2316. DOI: 10.1001/jama.2017.18718.

Subramanian A, Tamayo P, Mootha VK, Mukherjee S, Ebert BL, Gillette MA, Paulovich A, Pomeroy SL, Golub TR, Lander ES, Mesirov JP. 2005. Gene set enrichment analysis: a knowledge-based approach for interpreting genome-wide expression profiles. Proceedings of the National Academy of Sciences of the United States of America 102:15545-50. DOI: 10.1073/pnas.0506580102.

Sun L, Hui AM, Su Q, Vortmeyer A, Kotliarov Y, Pastorino S, Passaniti A, Menon J, Walling J, Bailey R, Rosenblum M, Mikkelsen T, Fine HA. 2006. Neuronal and glioma-derived stem cell factor induces angiogenesis within the brain. Cancer Cel 9:287-300. DOI:

10.1016/j.ccr.2006.03.003.

Tan Y, Zhang S, Xiao Q, Wang J, Zhao K, Liu W, Huang K, Tian W, Niu H, Lei T, Shu K. 2020. Prognostic significance of ARL9 and its methylation in low-grade glioma. Genomics 112:4808-4816. DOI: 10.1016/j.ygeno.2020.08.035.

Tang Z, Li C, Kang B, Gao G, Li C, Zhang Z. 2017. GEPIA: a web server for cancer and normal gene expression profiling and interactive analyses. Nucleic acids research 45:W98-W102. DOI: $10.1093 / \mathrm{nar} / \mathrm{gkx} 247$. 
436 Tsai CK, Huang LC, Tsai WC, Huang SM, Lee JT, Hueng DY. 2018. Overexpression of PLOD3 437 promotes tumor progression and poor prognosis in gliomas. Oncotarget 9:15705-15720.

438 DOI: 10.18632 /oncotarget.24594.

439 van den Bent MJ, Baumert B, Erridge SC, Vogelbaum MA, Nowak AK, Sanson M, Brandes AA, 440 Clement PM, Baurain JF, Mason WP, Wheeler H, Chinot OL, Gill S, Griffin M, Brachman

441 DG, Taal W, Rudà R, Weller M, McBain C, Reijneveld J, Enting RH, Weber DC, Lesimple T, Clenton S, Gijtenbeek A, Pascoe S, Herrlinger U, Hau P, Dhermain F, van Heuvel I, Stupp R, Aldape K, Jenkins RB, Dubbink HJ, Dinjens WNM, Wesseling P, Nuyens S, Golfinopoulos V, Gorlia T, Wick W, Kros JM. 2017. Interim results from the CATNON trial (EORTC study 26053-22054) of treatment with concurrent and adjuvant temozolomide for 1p/19q non-co-deleted anaplastic glioma: a phase 3, randomised, open-label intergroup study. Lancet 390:1645-1653. DOI: 10.1016/S0140-6736(17)31442-3.

Wang Y, Qian T, You G, Peng X, Chen C, You Y, Yao K, Wu C, Ma J, Sha Z, Wang S, Jiang T. 2015. Localizing seizure-susceptible brain regions associated with low-grade gliomas using voxel-based lesion-symptom mapping. Neuro-oncology 17:282-8. DOI: 10.1093/neuonc/nou130.

Wu X, Xiang H, Cong W, Yang H, Zhang G, Wang Y, Guo Z, Shen Y, Chen B. 2020. PLOD1, a target of miR-34c, contributes to cell growth and metastasis via repressing LATS1 phosphorylation and inactivating Hippo pathway in osteosarcoma. Biochemical and biophysical research communications 527:29-36. DOI: 10.1016/j.bbrc.2020.04.052.

Yamada Y, Kato M, Arai T, Sanada H, Uchida A, Misono S, Sakamoto S, Komiya A, Ichikawa T, Seki N. 2019. Aberrantly expressed PLOD1 promotes cancer aggressiveness in bladder cancer: a potential prognostic marker and therapeutic target. Molecular oncology 13:18981912. DOI: $10.1002 / 1878-0261.12532$.

Zhao Z, Meng F, Wang W, Wang Z, Zhang C, Jiang T. 2017. Comprehensive RNA-seq transcriptomic profiling in the malignant progression of gliomas. Scientific data 4:170024. DOI: 10.1038/sdata.2017.24.

Zhou Y, Zhou B, Pache L, Chang M, Khodabakhshi AH, Tanaseichuk O, Benner C, Chanda SK. 2019. Metascape provides a biologist-oriented resource for the analysis of systems-level datasets. Nature communications 10:1523. DOI: 10.1038/s41467-019-09234-6. 


\section{Table $\mathbf{1}$ (on next page)}

The characteristics of the public database samples

Notes: Abbreviations: CGGA, Chinese Glioma Genome Atlas; TCGA, The Cancer Genome Atlas; IDH, Isocitrate Dehydrogenase; MGMT, 06-methylguanine-DNA methyltransferase. 


\begin{tabular}{|c|c|c|c|c|}
\hline \multirow{2}{*}{ Characteristics } & \multicolumn{2}{|c|}{ CGGA(n=636) } & \multicolumn{2}{|c|}{ TCGA(n=592) } \\
\hline & case & proportion & case & proportion \\
\hline \multicolumn{5}{|l|}{ WHO Grade } \\
\hline II & 164 & $25.8 \%$ & 211 & $35.6 \%$ \\
\hline III & 207 & $32.5 \%$ & 238 & $40.2 \%$ \\
\hline IV & 265 & $41.7 \%$ & 143 & $24.2 \%$ \\
\hline \multicolumn{5}{|l|}{ Age(years) } \\
\hline$\geq 42$ & 342 & $53.8 \%$ & 349 & $59.0 \%$ \\
\hline$<42$ & 294 & $46.2 \%$ & 243 & $41.0 \%$ \\
\hline \multicolumn{5}{|l|}{ Gender } \\
\hline Male & 371 & $58.3 \%$ & 344 & $58.1 \%$ \\
\hline Female & 265 & $41.7 \%$ & 248 & $41.9 \%$ \\
\hline \multicolumn{5}{|l|}{ IDH mutation } \\
\hline Yes & 336 & $52.8 \%$ & 372 & $62.8 \%$ \\
\hline No & 300 & $47.2 \%$ & 220 & $37.2 \%$ \\
\hline \multicolumn{5}{|l|}{ 1p19q codeletion } \\
\hline Yes & 126 & $19.8 \%$ & 149 & $25.2 \%$ \\
\hline No & 510 & $80.2 \%$ & 443 & $74.8 \%$ \\
\hline \multicolumn{5}{|l|}{ MGMTp } \\
\hline \multicolumn{5}{|l|}{ methylation } \\
\hline Yes & 347 & $54.6 \%$ & & \\
\hline No & 289 & $43.4 \%$ & & \\
\hline \multicolumn{5}{|l|}{ Radio } \\
\hline Yes & 501 & $78.8 \%$ & & \\
\hline No & 135 & $21.2 \%$ & & \\
\hline \multicolumn{5}{|l|}{ Chemo } \\
\hline Yes & 465 & $73.1 \%$ & & \\
\hline No & 171 & $26.9 \%$ & & \\
\hline
\end{tabular}

2 Notes: Abbreviations: CGGA, Chinese Glioma Genome Atlas; TCGA, The Cancer Genome Atlas;

3 IDH, Isocitrate Dehydrogenase; MGMT, O6-methylguanine-DNA methyltransferase. 
Figure 1

Workflow of the whole study

Notes: Abbreviations: CGGA, Chinese Glioma Genome Atlas; TCGA, The Cancer Genome Atlas; GSE, GEO Series; GEO, Gene Expression Omnibus; HPA, The Human Protein Atlas; ROC, Receiver Operator Characteristic; DEGs, Differentially Expressed Genes; GSEA, Gene Set Enrichment Analysis; ECM, Extracellular Matrix.

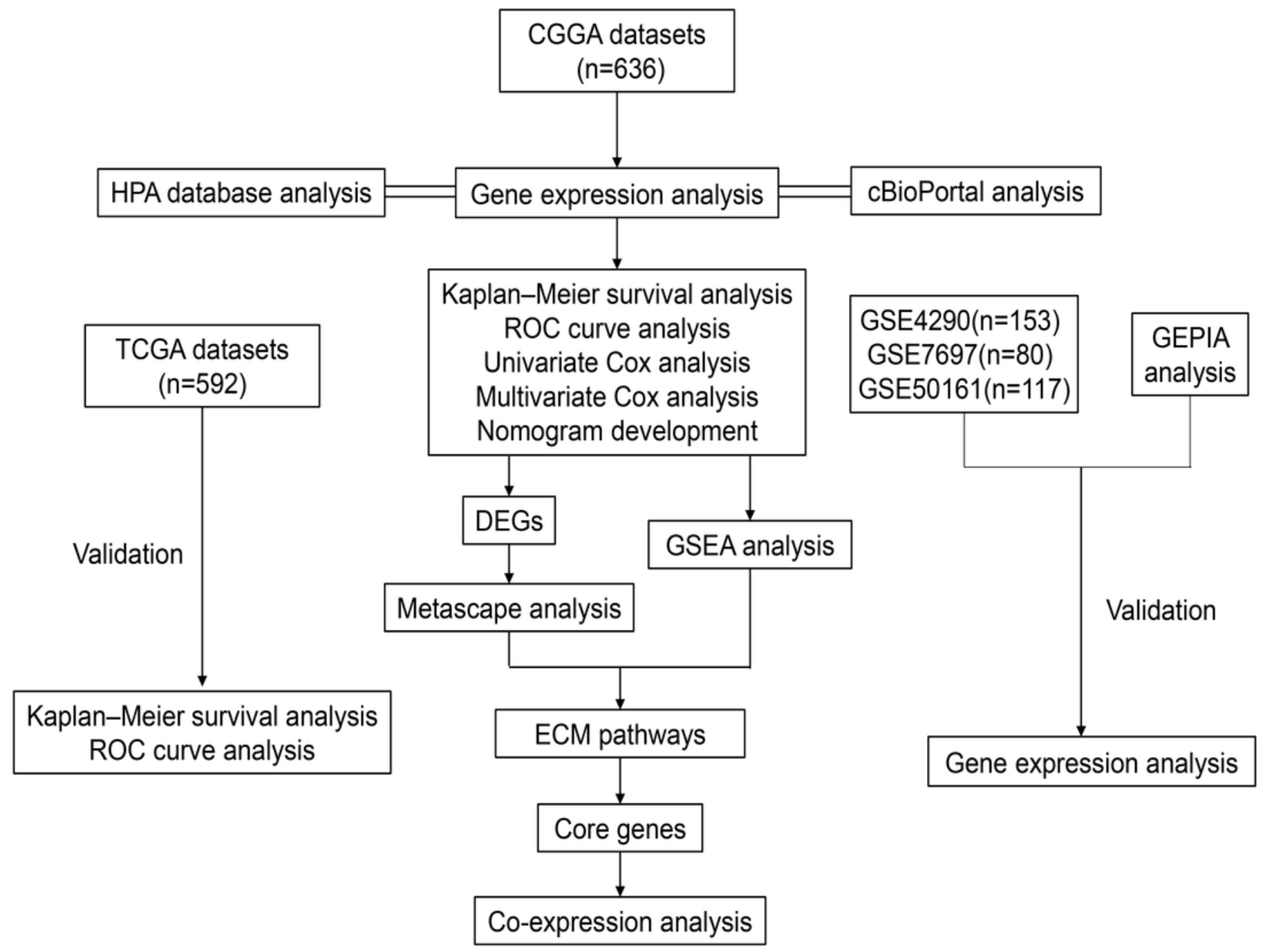




\section{Figure 2}

PLOD1 gene expression in glioma and normal tissue.

Figure 2: PLOD1 gene expression in glioma and normal tissue. (A) Comparison of PLOD1 expression levels between glioma and normal tissue in CGGA datasets. (B) GSE4290 dataset. (C) GSE7696 dataset. (D) GSE50161 dataset. (E) PLOD1 expression levels of GBM and LGG in GEPIA analysis, comparing to normal tissue respectively. (F) The genomic alteration of PLOD1 using cBioPortal online analysis. (G) Mutation types of PLOD1. (H) PLOD1 expression level across various cancer cell lines, including glioma cell lines (rank 4th, indicated by red boxes) from the CCLE database (Y-axis represents the expression level of PLOD1 in different cancer cell lines). 
A

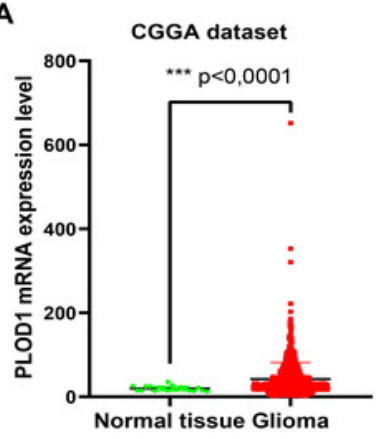

E
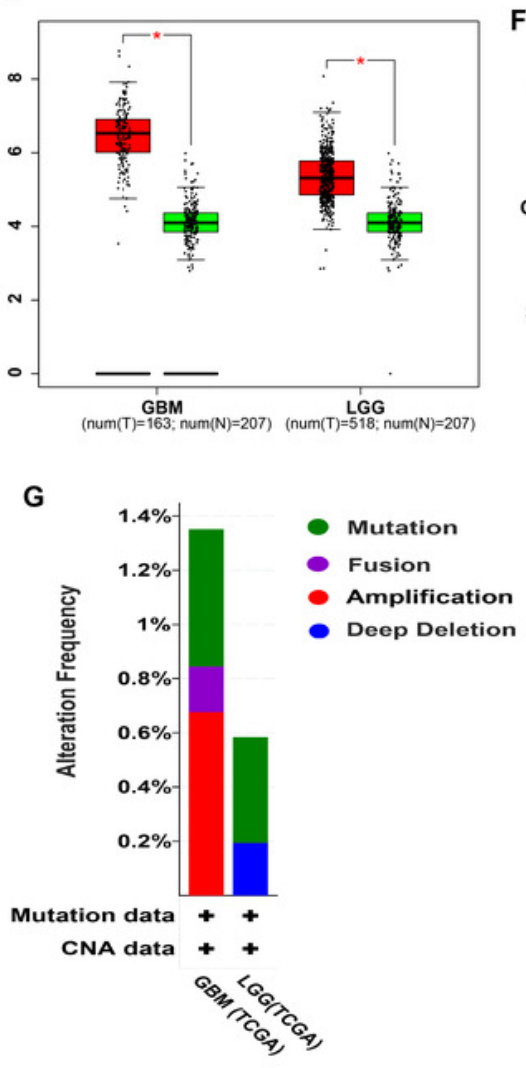

B

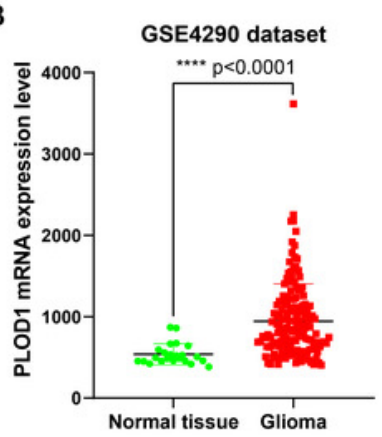

$\mathbf{F}$ c

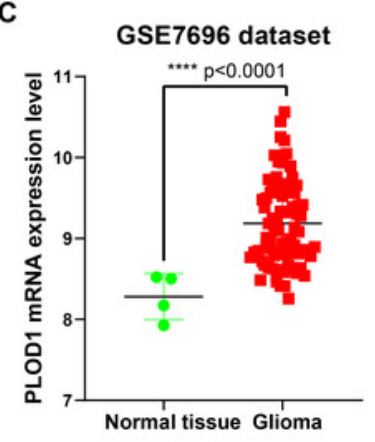

D

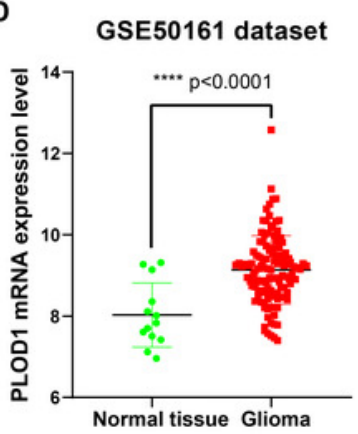

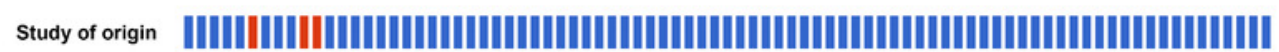

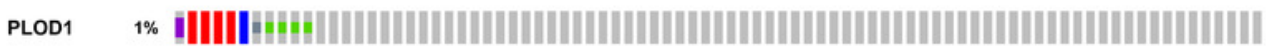
Genetic Alteration Missense Mutation (unknown significance) 플 No alterations Deep Deletion (unknown significance) \Amplification (unknown significance) Study of origin \Brain Lower Grade Glioma (TCGA, PanCancer Atlas) \Glioblastoma Multiforme (TCGA, PanCancer Atlas)

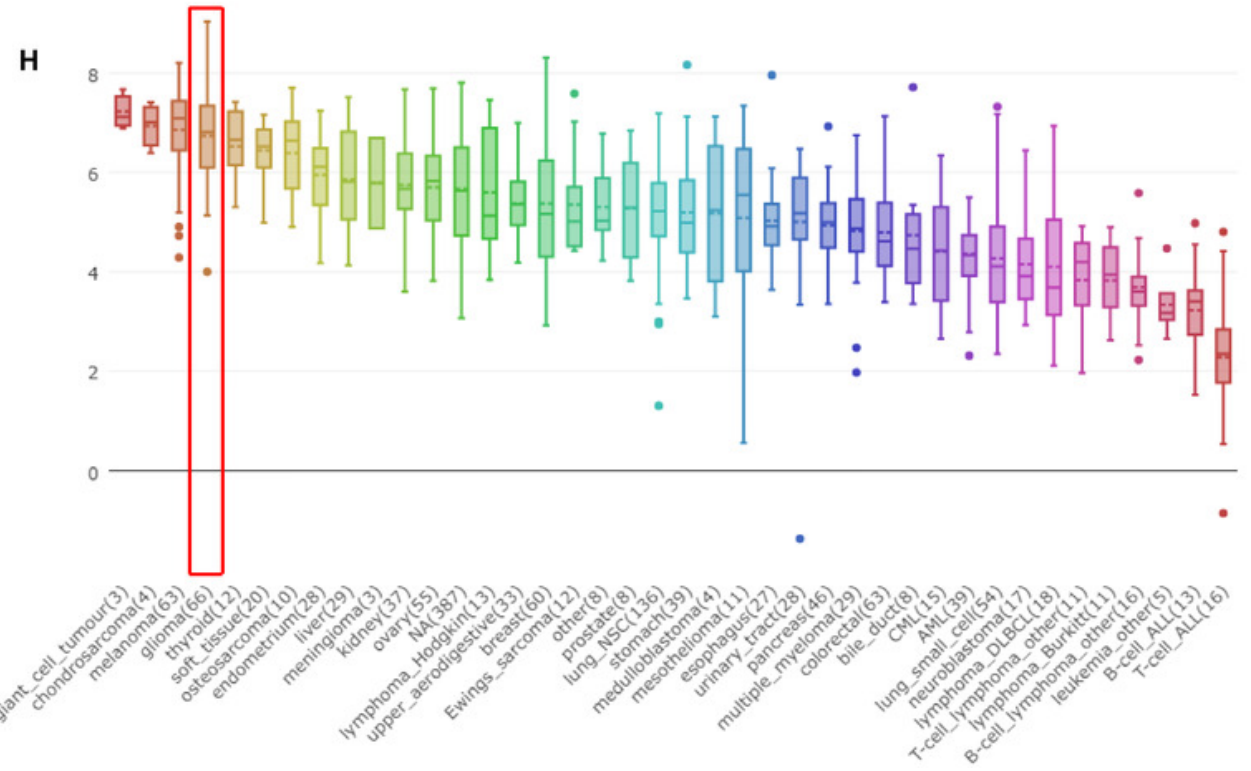




\section{Figure 3}

Survival analysis and prognostic values of PLOD1.

Survival analysis of glioma patients in the high PLOD1 and low PLOD1 groups. Red lines represent high expression and blue lines represent low expression. (A) Based on CGGA datasets. (B) Based on TCGA datasets. (C) Relationship between PLODI expression and IDH status on glioma survival. Green line represents low expression and IDH wildtype, red line represents high expression and IDH wildtype, blue line represents low expression and IDH mutation, purple line represents high expression and IDH mutation. (D) Relationship between PLOD1 expression and MGMT promoter methylation status on glioma survival. Blue line represents low expression and MGMT promoter methylation, green line represents high expression and MGMT promoter methylation, purple line represents low expression and MGMT promoter unmethylation, red line represents high expression and MGMT promoter unmethylation. Receiver operator characteristic curve analysis of PLOD1. Red lines represent 5 year survival, green lines represent 3 year survival and blue lines represent one 1 survival. (E) Based on CGGA datasets. (F) Based on TCGA datasets. 
PLOD1 level + high + low
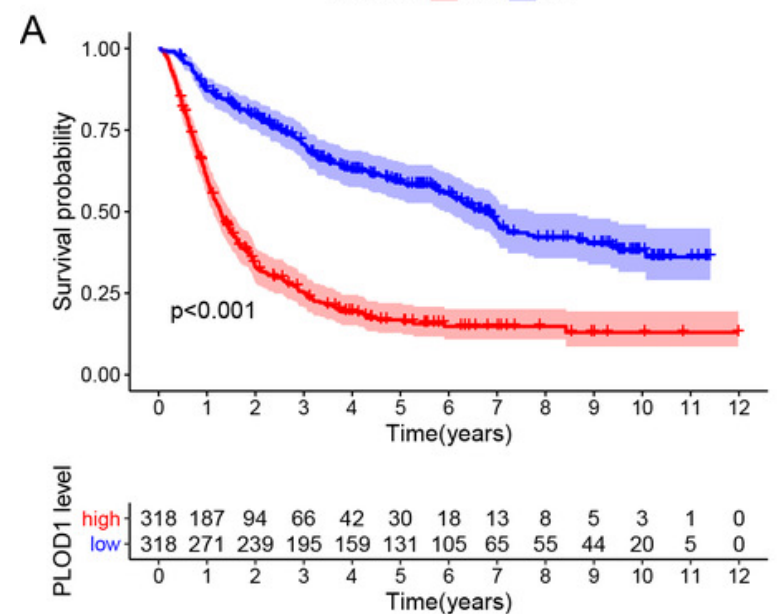

C

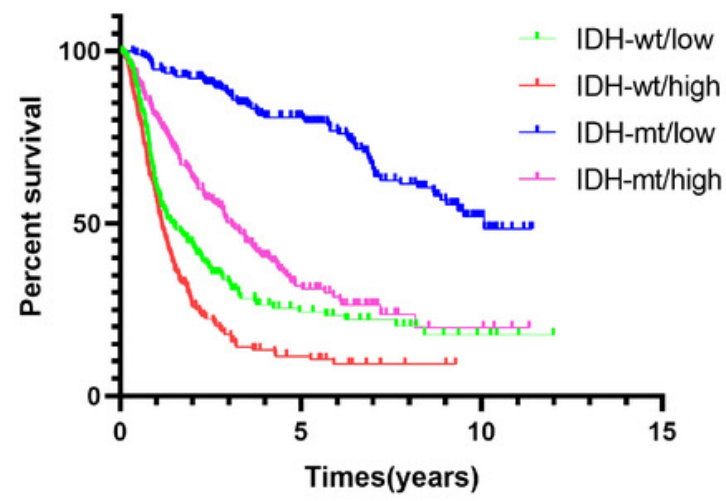

E

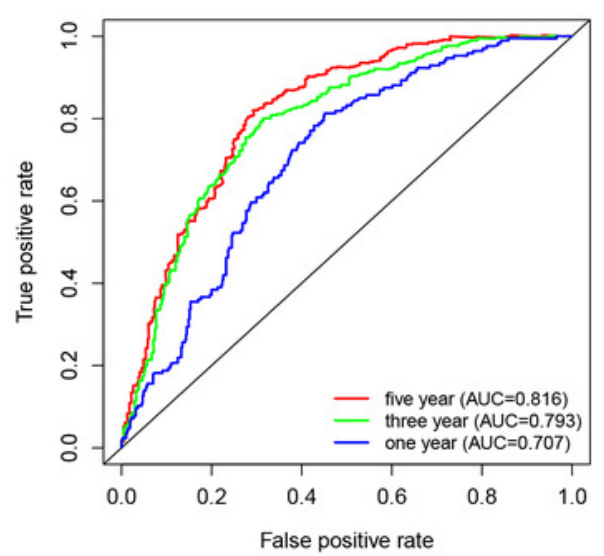

PLOD1 level thigh t low
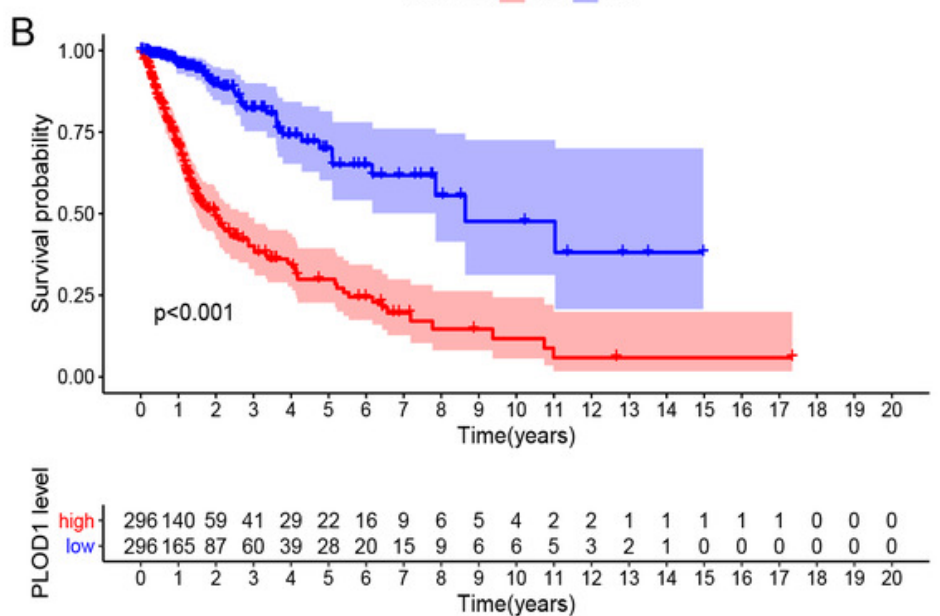

D

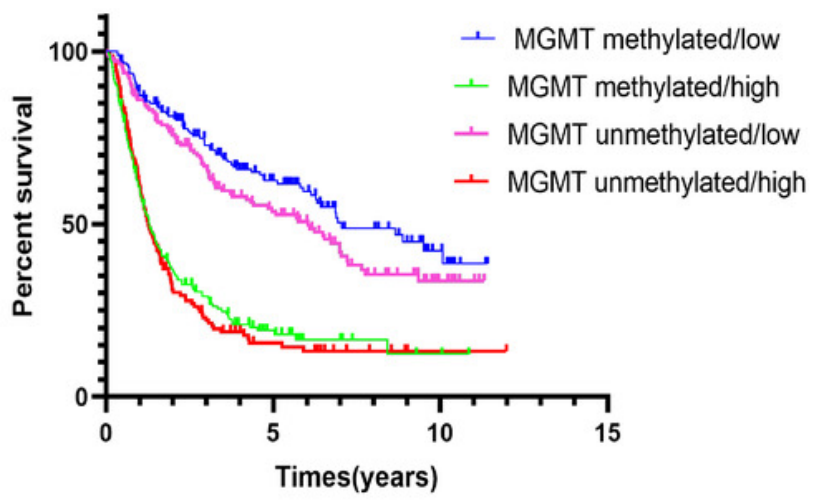

$\mathrm{F}$

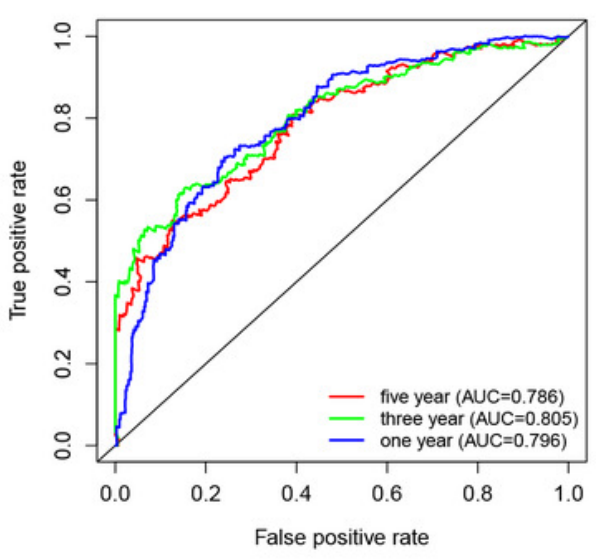




\section{Figure 4}

Independent prognostic analysis and development of nomogram of PLOD1 in glioma.

(A) Univariate analysis of PLOD1 based on CGGA datasets. (B) Multivariate analysis of PLOD1 based on CGGA datasets. (C) Prognostic nomogram to predict the survival of glioma patients based on the CGGA datasets. The values of grade, IDH, MGMT, 1p19q and PLODI are acquired from each variable axis. The total points on the axis are the sum values of these factors, which can predict the 1-, 2-, and 3-year survival. Calibration curves of the nomogram for predicting survival at 1, 2, and 3 years in the CGGA training cohort (D-F). 
A

$\begin{array}{lrr} & \text { pvalue } & \begin{array}{r}\text { Hazard ratio } \\ \text { PLOD1 }\end{array} \\ \text { PRS_type } & <0.001 & 1.986(1.796-2.198) \\ \text { Grade } & <0.001 & 2.188(1.857-2.579) \\ \text { Gender } & 0.545 & 2.857(2.473-3.300) \\ \text { Age } & <0.001 & 1.063(0.872-1.296) \\ \text { Radio } & 0.440 & 1.528(1.248-1.872) \\ \text { Chemo } & 0.064 & 1.238(0.988-1.553) \\ \text { IDH_mutation } & <0.001 & 0.322(0.262-0.395) \\ \text { 1p19q_codeletion } & <0.001 & 0.229(0.164-0.320) \\ \text { MGMTP_methylation } 0.155 & 0.868(0.714-1.055)\end{array}$

C

Points

Grade

IDH

MGMT

$1 \mathrm{p} 19 \mathrm{q}$

PLOD1

Total Points

1-year survival

2-year survival

3-year survival

D

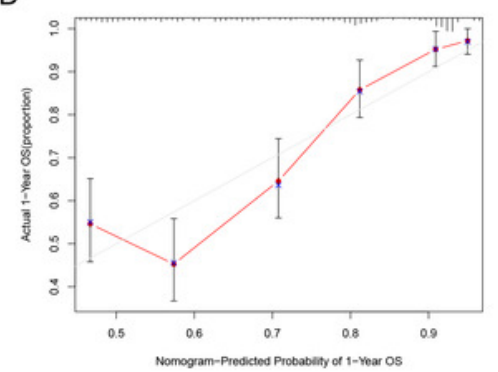

B

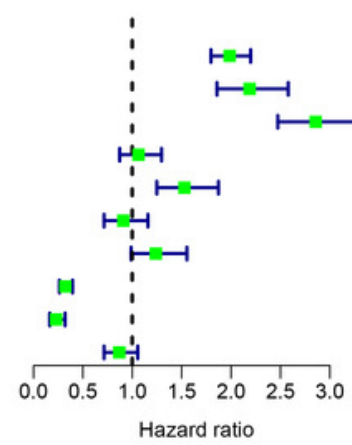

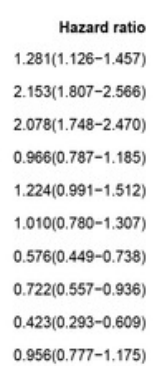

$0.956(0.777-1.175)$

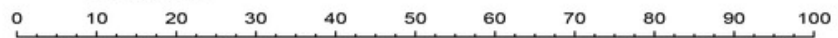
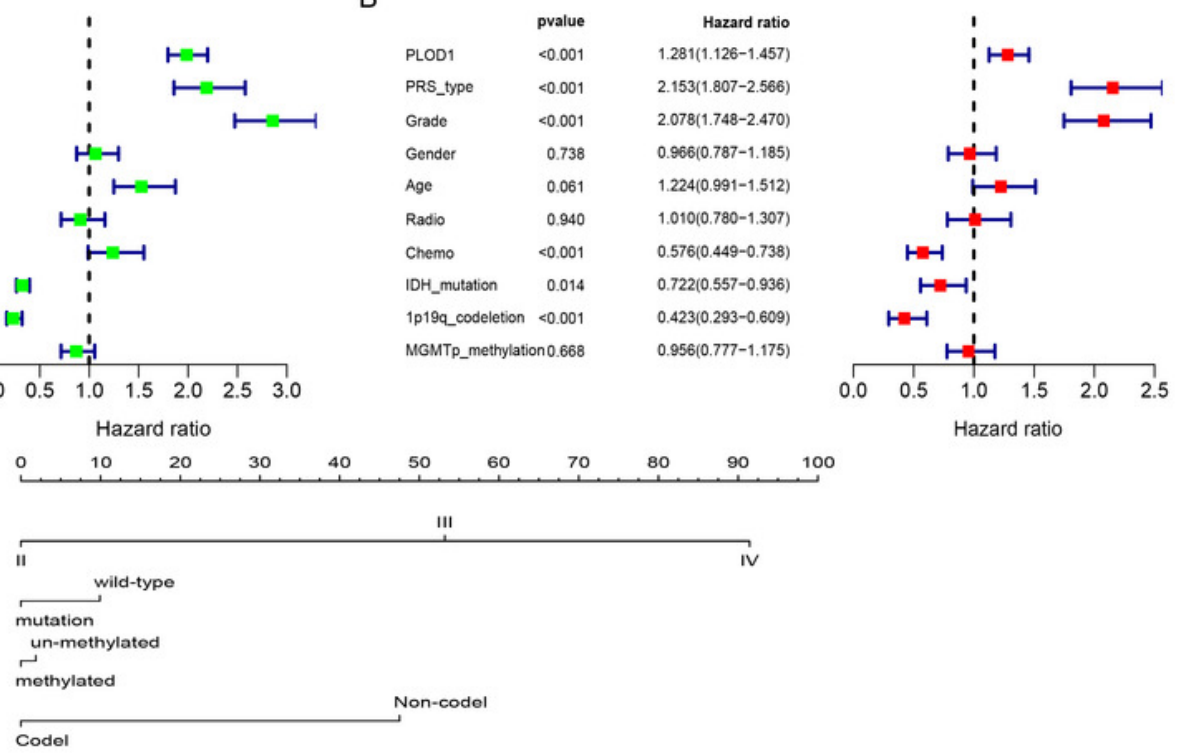

$\begin{array}{lllllllllllll}2.5 & 3 & 3.5 & 4 & 4.5 & 5 & 5.5 & 6 & 6.5 & 7 & 7.5 & 8 & 8.5\end{array}$

\begin{tabular}{llllllllllllllllll}
\hline 0 & 20 & 40 & 60 & 80 & 100 & 120 & 140 & 160 & 180 & 200 & 220 & 240 & 260 & 280
\end{tabular}

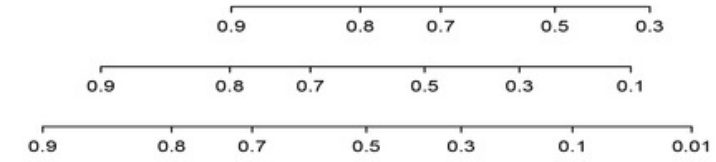

E

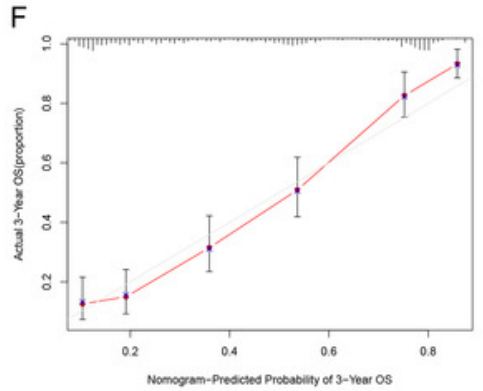


Figure 5

Correlation analysis with clinicopathologic characteristics of PLOD1 in glioma.

(A) age (<42, n=294; $\geq 42, n=342)$, (B) grade (WHO II, $n=164 ;$ WHO III, $n=207$; WHO IV, $n=265)$, (C) PRS type (Primary, $n=433$; Recurrent, $n=179$; Secondary, $n=24)$, (D) 1p19q codeletion status (Codel, $n=126$; Non-codel, $n=510$ ), and (E) IDH mutation status (Mutant, $n=347 ;$ Wildtype, $n=289$ ).
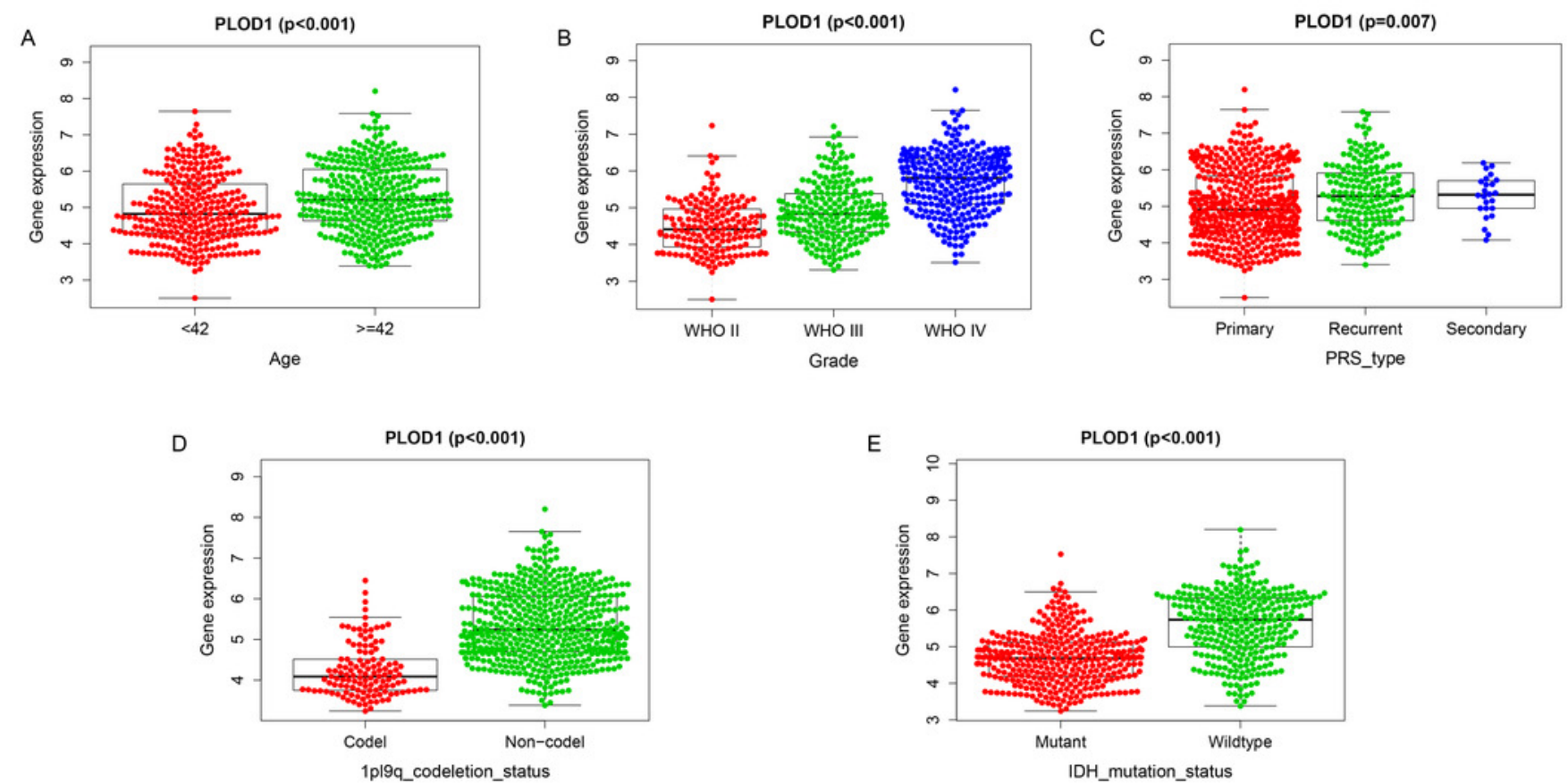


\section{Figure 6}

DEGs and enrichment pathways analysis of PLOD1.

(A) Volcano plot of differentially expressed genes. Red nodes represent the significantly upregulated genes with $\log F C>2$ and $p<0.05$. Green nodes represent the significantly downregulated genes with $\log F C<-2$ and $p<0.05$. (B) Heatmap of enriched terms across DEGs in Metascape analysis. (C) Enrichment plot of ECM receptor interaction pathway from GSEA. (D) The Venn diagram of the common genes, which is obtained by intersecting the core genes of the ECM pathway with DEGs.

A

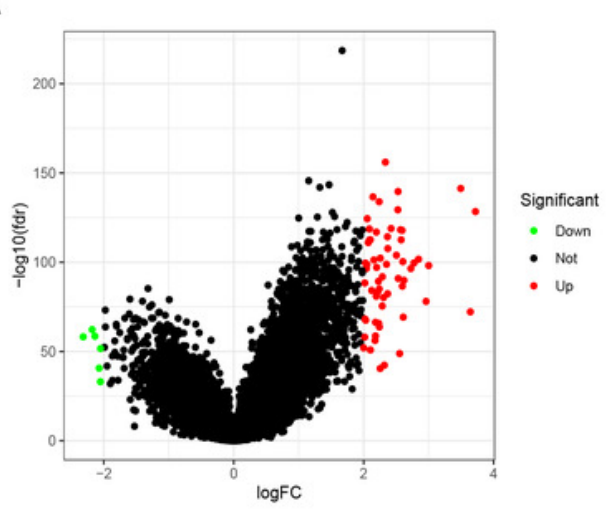

C Enrichment plot: KEGG_ECM_RECEPTOR_INTERACTION

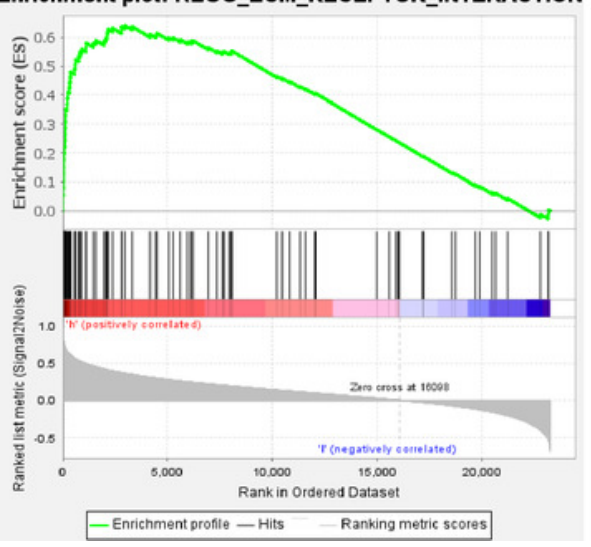

B

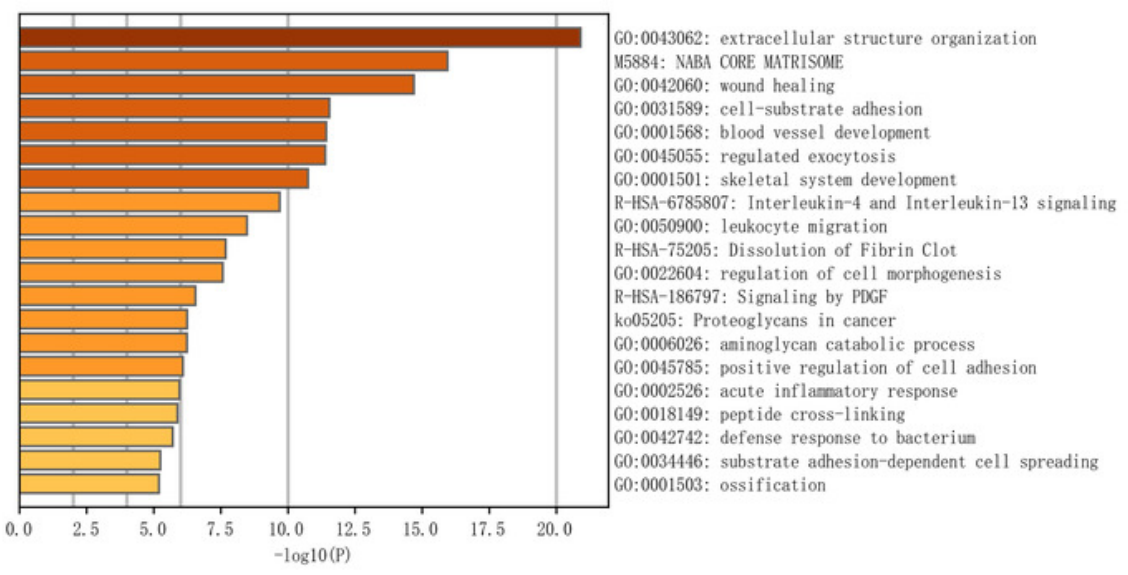

D

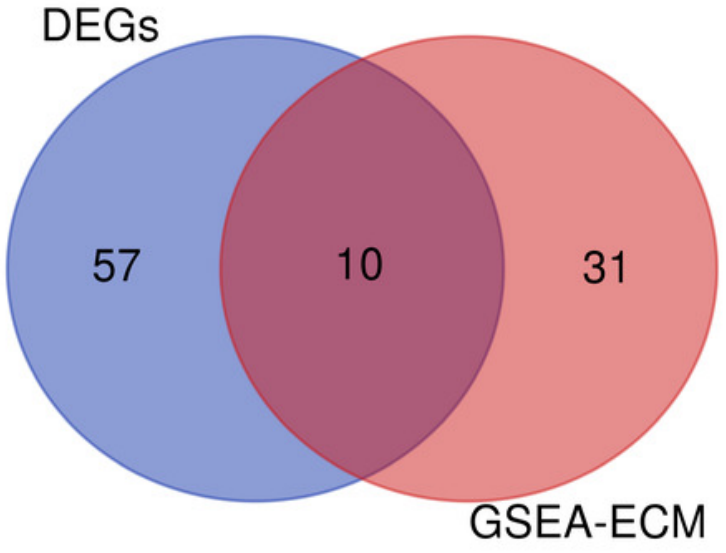


Figure 7

Co-expression analysis of PLOD1 with common genes.

(A) CD44; (B) COL1A1; (C) COL1A2; (D) COL3A1; (E) COL4A1; (F) COL4A2; (G) COL6A2; (H) FN1; (I) HSPG2; (J) SPP1. (K) Circular plot of common genes with PLOD1. Red represents positive association. (L) Heatmap of the common genes with PLOD1.
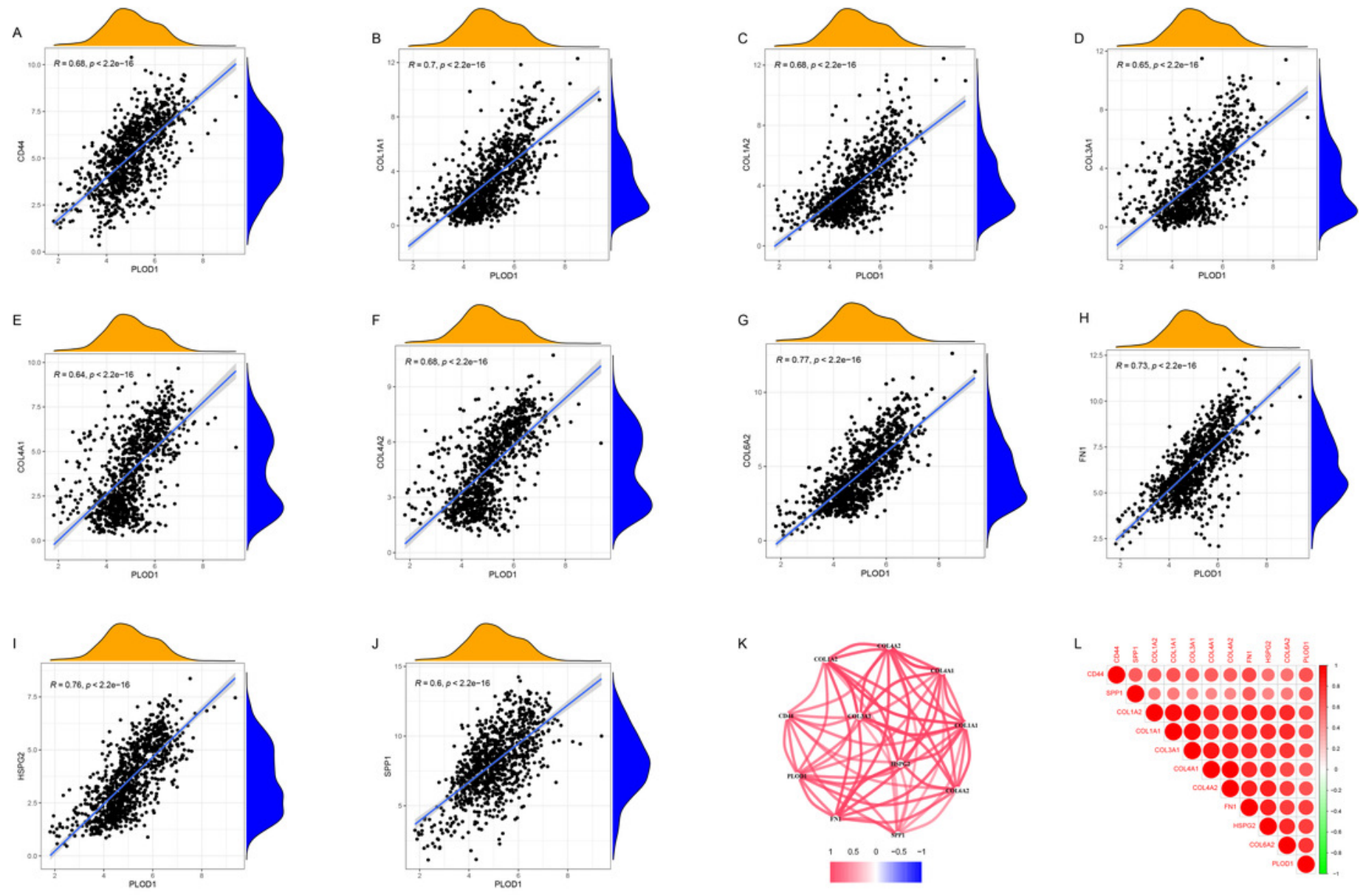
Figure 8

Common genes expression levels in glioma and correlations with survival in GEPIA analysis.

$(A, B)$ CD44; (C, D) COLIA1; (E, F) COLIA2; (G,H) COL3A1; (I, J) COL4A1; (K, L) COL4A2; (M, N) COL6A2; $(O, P)$ FN1; $(Q, R)$ HSPG2; $(S, T)$ SPP1. 

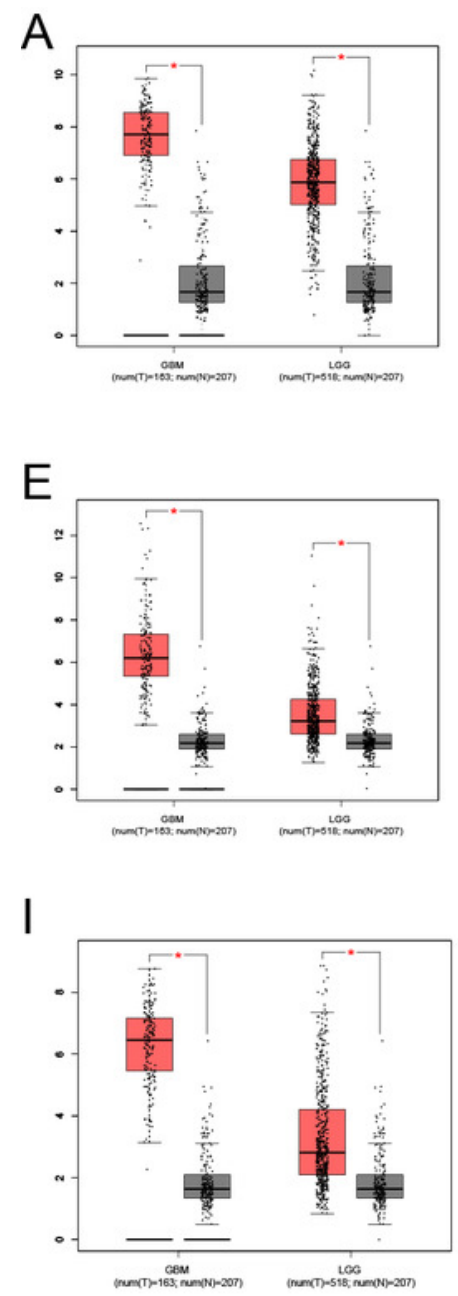

M
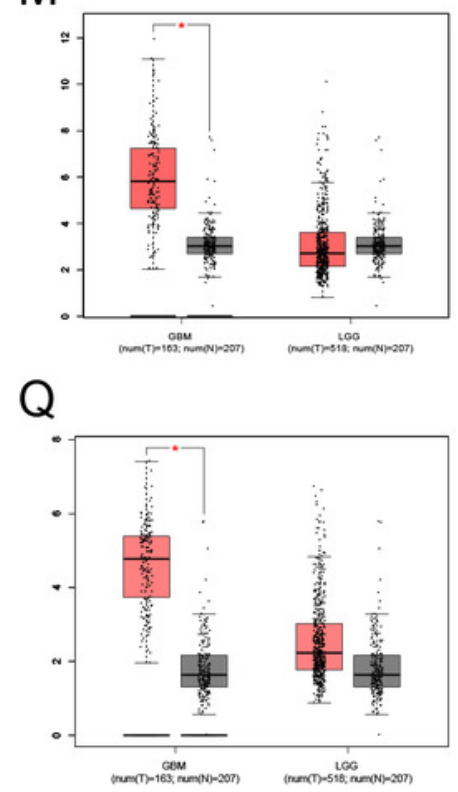
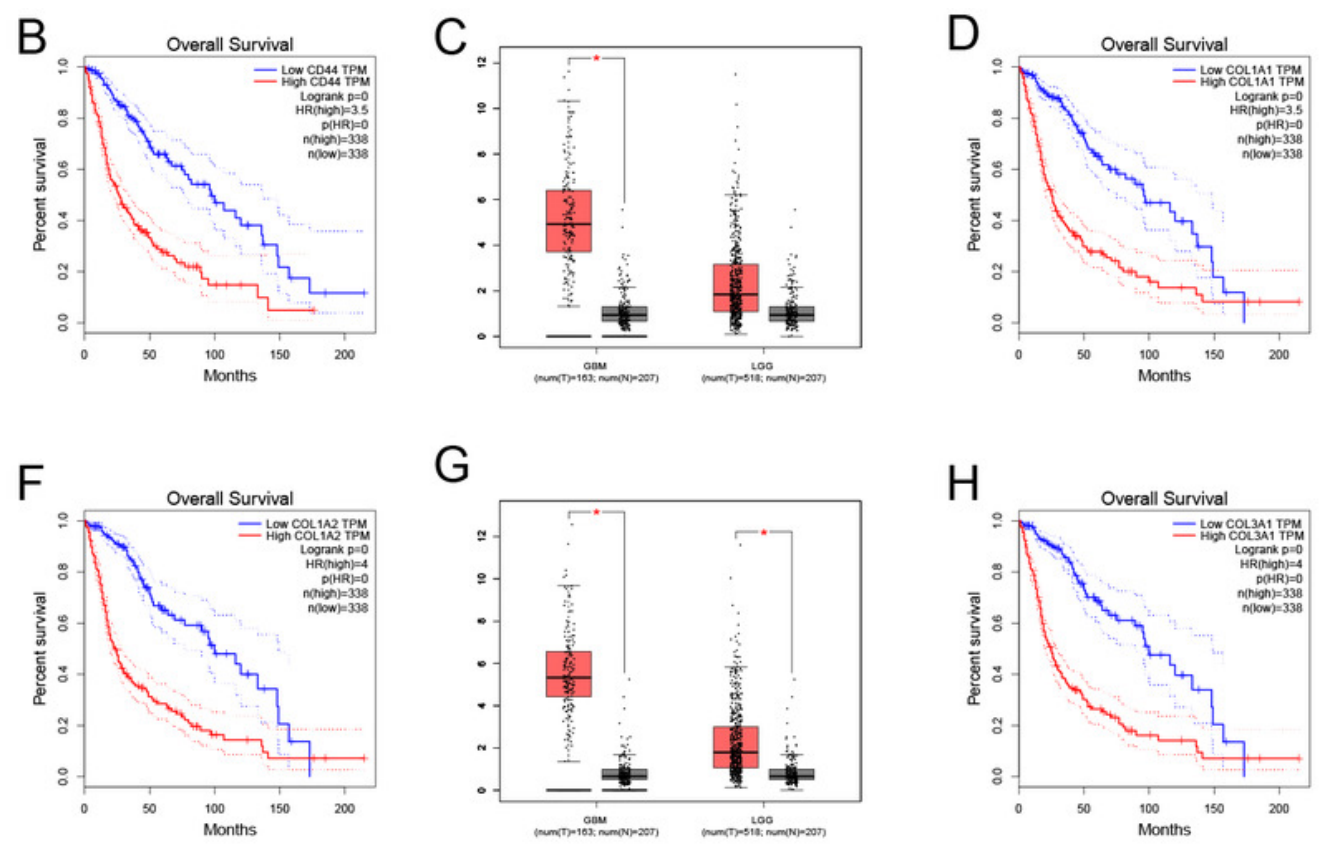

G
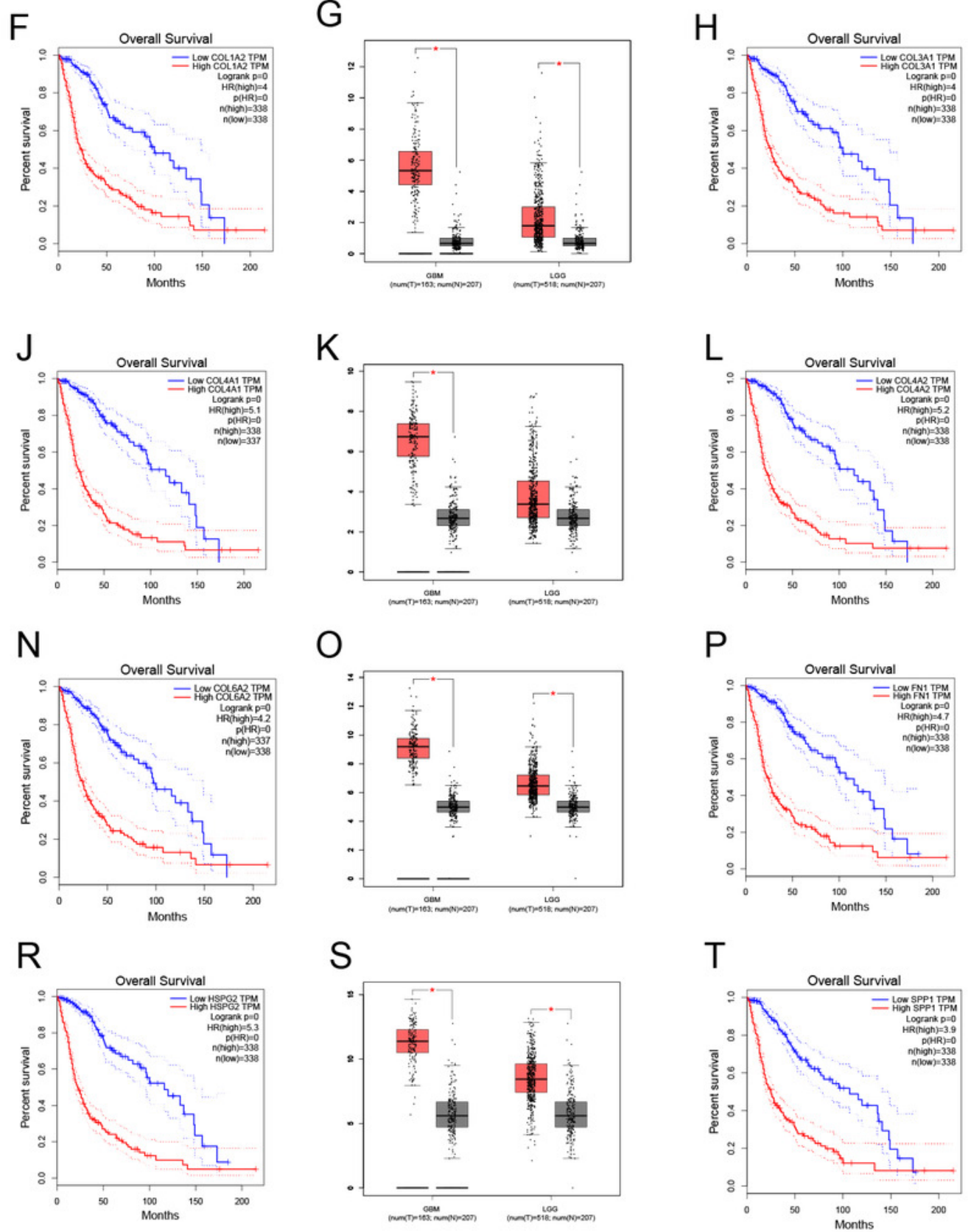

S

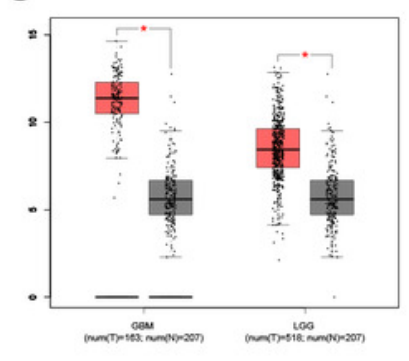

T

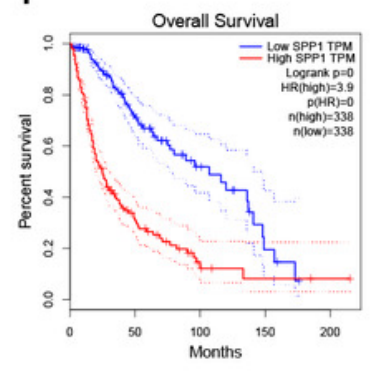

